Fortgeschrittene diabetische Nephropathie

\title{
Antioxidativer Wirkstoff schützt offenbar die Nierenfunktion
}

Pergola PE, Raskin P, Toto RD et al. BEAM

Study Investigators. Bardoxolone methyl and kidney

function in CKD with type 2 diabe-

tes. N Engl J Med 2011;365(4):327-36.
Fragestellung: Schützt Bardoxolon-Methyl die Nierenfunktion bei Patienten mit Typ-2-Diabetes und chronischem Nierenversagen?

Einleitung: Die diabetische Nephropathie führt häufig zum chronischen Nierenversagen (CKD). Entzündung und oxidativer Stress tragen beide zur Krankheitsprogression bei. Bardoxolon-Methyl ist ein oraler antioxidativer Wirkstoff, der die Entzündung moduliert. Kurzzeitstudien mit dieser Substanz zeigten eine positive Wirkung auf die CKD bei Patienten mit Typ-2-Diabetes. Langzeitstudien gab es bisher nicht.

Methodik: In der doppelblinden randomisierten placebokontrollierten Phase-II-Studie wurden 227 Patienten mit Typ-2-Diabetes und moderater oder schwerer CKD (definiert als GFR von $20-45 \mathrm{ml} / \mathrm{min}$ ) pro 1,72 m2 Körperoberfläche) aufgenommen. Im 1:1:1:1 Verhältnis wurden Placebo oder 25, 75 oder 150 Bardoxolon-Methyl einmal täglich eingenommen. Primärer Endpunkt war die Veränderung der geschätzten GFR nach 24 Wochen im Vergleich zum Studienstart. Sekundärer Endpunkt war die GFR-Veränderung nach 1 Jahr.

Ergebnisse: Patienten, die Bardoxolon-Methyl erhielten, hatten nach 24 Wochen einen signifikanten Anstieg der Mittleren ( \pm SD) GFR im Vergleich zu Placebo (GFR-Veränderungen in den einzelnen Gruppen von $8,2 \pm 1,5 \mathrm{ml}$ in der 25-mg Gruppe, $11,4 \pm 1,5 \mathrm{ml}$ in der 75-mg Gruppe, und 10,4 $\pm 1,5 \mathrm{ml}$ in der 150-mg Gruppe; $\mathrm{p}<0,001)$. Diese Verbesserung der GFR war auch noch nach 52 Wochen signifikant $(5,8 \pm 1,8 \mathrm{ml}, 10,5 \pm 1,8 \mathrm{ml}$, und 9,3 $\pm 1,9 \mathrm{ml}$ ). Milde, dosisabhängige Muskelkrämpfe waren die häufigsten unerwünschten Nebenwirkungen in der Verumgruppe. Hypomagnesiämie, leichte Anstiege von ALT (GPT) und gastrointestinale Nebenwirkungen waren häufiger in den Gruppen, die Bardoxolon-Methyl erhalten hatten.

Schlussfolgerungen: Bardoxolon-Methyl führte nach 24 Wochen zu einer Verbesserung der geschätzten GFR bei Patienten mit fortgeschrittener Nierenschädigung und Typ-2-Diabetes. Die Verbesserung zeigte sich auch nach 1 Jahr noch, sodass Bardoxolon-Methyl ein neues Medikament bei Patienten mit CKD werden könnte.

Kommentar: Die längerfristige Verbesserung der GFR bei Patienten mit chronischer Niereninsuffizienz ist ein vielversprechendes Ergebnis. Bardoxolon-Methyl ist eine synthetisches Triterpenoid, das zu der Gruppe der Antioxidant inflammatory Modulators (AIM) gehört und ein potenter Induktor der Nrf2 Pathways ist. Es bleibt zu hoffen, dass es in Folgestudien den hohen Erwartungen gerecht werden kann.

PD Dr. med. Nanette C. Schloot, Düsseldorf

\section{Risikoprädiktion Typ-2-Diabetes}

\section{Schwach mit $\mathrm{HbA}_{1 \mathrm{c}}$ allein, stark plus Einstunden-Plasmaglukose}

\footnotetext{
Abdul-Ghani MA, Abdul-Ghani T, Müller G et al. Role of Glycated Hemoglobin in the Prediction of Future Risk of T2DM. J Clin Endocrinol Metab 2011;96(8):2596-600
}

Fragestellung: Wie potent ist eine $\mathrm{HbA}_{1 \mathrm{c}}$-Messung, um das Risiko für zukünftigen Diabetes zu Erkennen?

Hintergrund: Der $\mathrm{HbA}_{1 \mathrm{c}}$ wird als Diagnose- und Screeninginstrument für einen Diabetes mellitus derzeit heftig diskutiert. Ein $\mathrm{HbA}_{1 \mathrm{c}}$-Wert von über 6,5 bestimmt diagnostisch valide einen Diabetes mellitus. Der Bereich zwischen 5,7 und 6,5 wird kontrovers diskutiert. Un- klarheit herrscht über die Bedeutung eines $\mathrm{HbA}_{1 \mathrm{c}}$-Wertes unter 5,7\%. Eine weitere Diskussion gibt es bezüglich der diagnostischen Qualität des oralen Glukose-Toleranz-Tests (OGTT). Dabei besteht eine unterschiedliche Wertigkeit für die Nüchternglukose, und Zweistundenglukose. Immer wieder diskutiert wird allerdings auch die Einstundenglukose und ihre Wertigkeit zur Diabetesprädiktion. 